\title{
Physical modeling of echelle spectrographs: the CARMENES case study
}

Pinto, M. Tala, Chanumolu, A., Quirrenbach, A., Reffert, S., Zechmeister, M., et al.

M. Tala Pinto, A. Chanumolu, A. Quirrenbach, S. Reffert, M. Zechmeister, F. Bauer, "Physical modeling of echelle spectrographs: the CARMENES case study," Proc. SPIE 10705, Modeling, Systems Engineering, and Project Management for Astronomy VIII, 107051 U (10 July 2018); doi: $10.1117 / 12.2313211$

Event: SPIE Astronomical Telescopes + Instrumentation, 2018, Austin, Texas, United States 


\title{
Physical modeling of echelle spectrographs: the CARMENES case study
}

\author{
M. Tala Pinto ${ }^{\mathrm{a}}$, A. Chanumolu ${ }^{\mathrm{b}}$, A. Quirrenbach ${ }^{\mathrm{a}}$, S. Reffert ${ }^{\mathrm{a}}$, M. Zechmeister ${ }^{\mathrm{c}}$, and F. Bauer ${ }^{\mathrm{d}}$ \\ ${ }^{a}$ Landessternwarte, Zentrum für Astronomie der Universität Heidelberg, Königstuhl 12, 69117 \\ Heidelberg, Germany

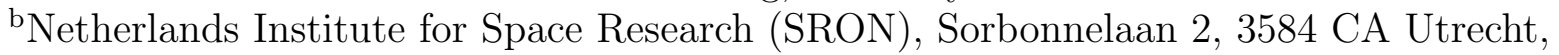 \\ Netherlands \\ 'Institut für Astrophysik (IAG), Georg-August-Universität Göttingen, Friedrich-Hund-Platz 1, \\ 37077 Göttingen, Germany \\ ${ }^{\mathrm{d} I n s t i t u t o ~ d e ~ A s t r o f i ́ s i c a ~ d e ~ A n d a l u c i ́ a ~(I A A-C S I C), ~ A p d o . ~ 3004, ~ E-18008 ~ G r a n a d a, ~ S p a i n ~}$
}

\begin{abstract}
We have developed a generic physical modeling scheme for high resolution spectroscopy based on simple optical principles. This model predicts the position of centroids for a given set of spectral features with high accuracy. It considers off-plane grating equations and rotations of the different optical elements in order to properly account for tilts in the spectral lines and order curvature. In this way any astronomical spectrograph can be modeled and controlled without the need of commercial ray tracing software. The computations are based on direct ray tracing applying exact corrections to certain surfaces types. This allows us to compute the position on the detector of any spectral feature with high reliability. The parameters of this model, which describe the physical properties of the spectrograph, are continuously optimized to ensure the best possible fit to the observed spectral line positions. We present the physical modeling of CARMENES as a case study. We show that our results are in agreement with commercial ray tracing software. The model prediction matches the observations at a pixel size level, providing an efficient tool in the design, construction and data reduction of high resolution spectrographs.
\end{abstract}

Keywords: hi-res spectroscopy, wavelength solution, physical modeling

\section{INTRODUCTION}

Along the way to high precision radial velocity (RV) measurements there have been many improvements regarding the design, construction and optimization of high resolution spectrographs. White pupil designs improved instruments efficiencies, optical fibers allowed us to detach instruments from the telescope focal plane and mount them in gravity controlled environments, multi-channel spectrographs increase the wavelength range observed, and thermal and pressure control are critical to achieve few $\mathrm{m} / \mathrm{s}$ RV precision when using the simultaneous calibration technique. Moreover, new calibration sources, such as etalons or laser frequency combs, are pushing the limit of the RV precision into the $\mathrm{cm} / \mathrm{s}$ regime. However, in contrast to the advancements in hardware, there has not been so much work on trying to transmit the information of the physical properties of the spectrograph to the instrument data reduction software.

In particular, when performing the wavelength calibration of a given set of spectra, the wavelength solution - the function that maps from pixel to wavelength space - is derived in a purely empirical way, by fitting polynomials to a sparse calibration line spectrum. One way to ensure that the engineering data propagates from instrument building to operations is to catch all the engineering information into a physical model-based description of the instrument and use this model to compute the wavelength solution.

The ESO group developed a model-based calibration tool ${ }^{1}$ which has been successfully applied to FOS $^{2}$ and STIS $^{3}$ on HST, as well as CRIRES ${ }^{4}$ and X-SHOOTER ${ }^{5}$ on the VLT. This model-based approach is limited to

Further author information: (Send correspondence to M.T.P.)

M.T.P.: E-mail: mtala@lsw.uni-heidelberg.de, Telephone: +49 6221541733

Modeling, Systems Engineering, and Project Management for Astronomy VIII

edited by George Z. Angeli, Philippe Dierickx, Proc. of SPIE Vol. 10705

$107051 \mathrm{U} \cdot$ (c) $2018 \mathrm{SPIE} \cdot \mathrm{CCC}$ code: $0277-786 \mathrm{X} / 18 / \$ 18$

Proc. of SPIE Vol. $10705107051 \mathrm{U}-1$ 
on-axis configuration of the optics with all aberration corrections applied together as a polynomial at the detector plane. ${ }^{4}$ Following the same philosophy, Chanumolu et al. $2015^{6}$ developed a physical model for the Hanle echelle spectrograph that provides the exact position of chief rays leaving only the centroid corrections, which are of the order of sub-pixel, at the detector plane. This modeling approach is based on paraxial ray-tracing including exact corrections for some surface types and Buchdahl aberration coefficients for complex modules such as camera systems. In addition, it is able to track the difference in the centroids between two fiber images across the spectrum, as well as instrumental shifts between two fibers, providing an efficient 1D order extraction tool for varying slit positions and orientations. ${ }^{7}$

Our modeling scheme is based on direct ray tracing of the chief ray from the slit to the detector plane, applying exact corrections when tracing through curved surfaces. The direct ray tracing provides an accurate theoretical description of the instrument, as opposed to paraxial ray tracing, as it calculates the exact the position of each photon along the optical path of the spectrograph.

In most cases hollow cathode lamp (HCL) spectra are used to derive the model parameters that match the observations. The optimized model can be used to understand instrument behavior, quality checks and trending. The model once matched with the observations can predict the centroid position of any HCL spectral line.

The modeling of CARMENES is done in parallel with science observations, including daily calibrations. At the time of writing of this paper, the CARMENES survey has been observing for two and a half years, providing a wide baseline of observations and daily calibrations.

In section 2 we describe briefly the CARMENES spectrograph and its main components, while in section 3 we describe the formalism of our modeling approach, and its comparison with ZEMAX, a commercial ray tracing software. In section 4 we present preliminary results of the performance of the model with real HCL observations. Finally, in section 5 we present our conclusions and plans for further work.

\section{CARMENES OPTICAL DESIGN}

The CARMENES instrument ${ }^{8}$ is a pair of high-resolution spectrographs covering the wavelength range between 0.52 to $1.71 \mu \mathrm{m}$, optimized for precision radial velocity measurements. It is installed at the $3.5 \mathrm{~m}$ telescope of the Calar Alto observatory in Spain, and it will survey $~ 300 \mathrm{M}$ dwarfs with the aim of detecting super-earths in the habitable zone. The visible channel covers the wavelength range between $0.52-0.96 \mu \mathrm{m}$ at a measured spectral resolution of $R=93,400$, while the near-infrared channel covers the wavelength range between $0.91-1.7 \mu \mathrm{m}$ at a measured spectral resolution of $R=82,000$. The entrance aperture on sky is 1.5 arcsec.

The optical design of both channels consists of a grism cross-dispersed, white-pupil echelle spectrograph working in quasi-Littrow configuration with a two-beam, two-slice image slicer. The VIS channel is housed in a thermally stabilized vacuum vessel at a pressure of $\sim 10^{-5} \mathrm{mbar}$ and at a temperature of $\sim 12^{\circ} \mathrm{C}$. The NIR channel is also housed in a vacuum vessel stabilized at a temperature of $140 \mathrm{~K}$.

Both channels are fed with two $100 \mu \mathrm{m}$ diameter octagonal fibers, mounted next to each other, with a $0.346 \mathrm{~mm}$ separation center-to-center, to provide a simultaneous reference for instrumental drift corrections. The separation between the fibers results in a minimum separation of 7 pixels between science and calibration pseudo slit projected on the detector.

Table 1 summarizes the main features of the VIS spectrograph. Fig. 1 shows the optical layout of the VIS channel.

The $\mathrm{F} / \mathrm{N}$ lens system converts the $\mathrm{F} / 3.5$ output beam of the fibers into the $\mathrm{F} / 10.2$ focal ratio of the collimator. The image slicer is placed at the focal plane of the $\mathrm{F} / \mathrm{N}$ system, where the image of the octagonal fibers are sliced and re-arranged into a pseudo slit. The output beam from the slicer is then projected onto the collimator. The collimator is a parabolic mirror. After the collimator, the beam is reflected to the echelle grating, and bounced back to the collimator. After the second pass on the collimator, the beam is focused into a flat folding mirror that reflects the beam back to the collimator for a third pass. The collimator reflects the beam to the cross-dispersing grism and then to the camera lens that images the spectrum in the detector plane.

The collimator, echelle grating and folding mirror are made of Zerodur, for low thermal expansion.

The optical design of both channels is similar. Their main differences are the coatings applied to the reflective surfaces -collimator, echelle grating and folding mirror-, the materials and properties of the refractive optical elements - F/N system, cross-dispersion grism and camera lens system - and the detector characteristics. In this 


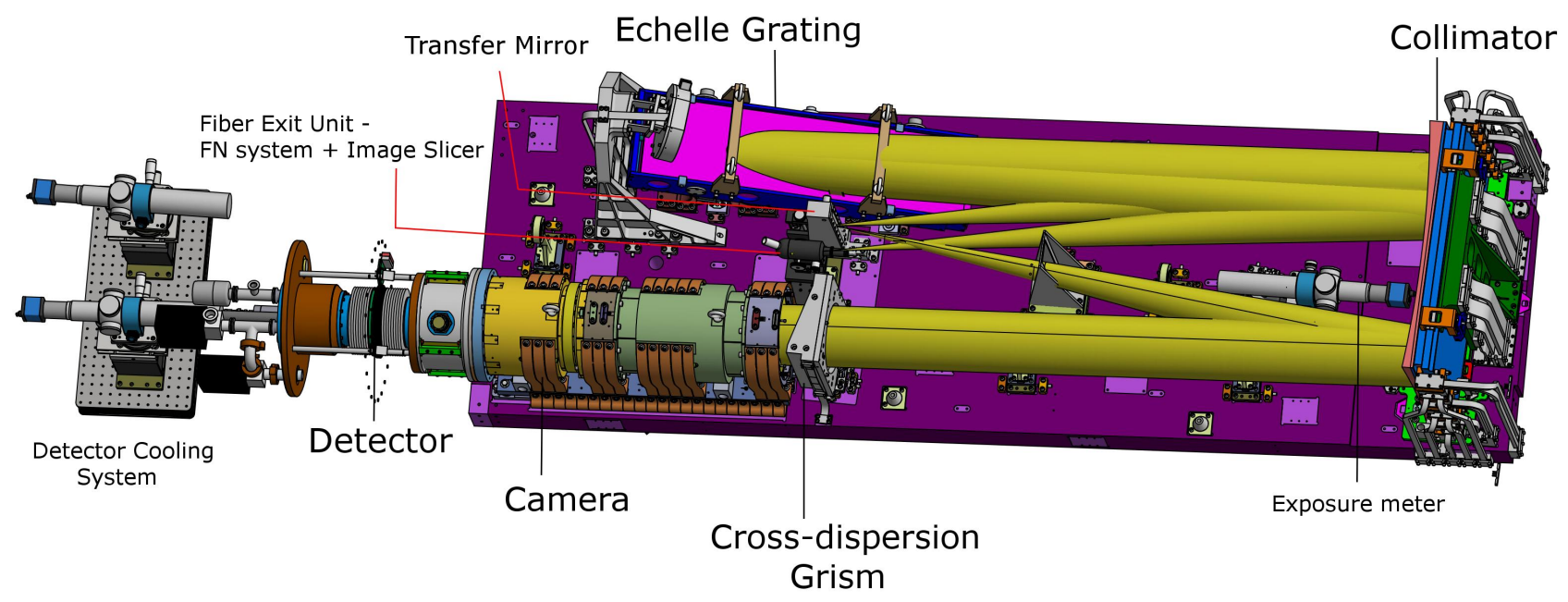

Figure 1. CARMENES VIS channel opto-mechanical layout. Coordinate system employed in the physical modeling is also shown.

paper we will refer to the modeling of the CARMENES VIS channel.

\begin{tabular}{|c|c|}
\hline Wavelength range & $550-1050 \mathrm{~nm}$, orders 53 to 116 \\
\hline Resolving power $(\lambda / \Delta \lambda)$ & 93,400 \\
\hline Fiber input & $100 \mu \mathrm{m}$ CeramOptec Optran WF $100 / 100 \mathrm{P}$ \\
\hline Collimator & $f=1590 \mathrm{~mm}$, material $=$ Zerodur \\
\hline Echelle grating & $\mathrm{R} 4,31.6$ grooves $/ \mathrm{mm}, 154 \mathrm{~mm} \times 596 \mathrm{~mm}$ \\
\hline Cross-disperser grism & $\mathrm{LF} 5$, Apex $=17.8 \mathrm{deg}, 223$ grooves $/ \mathrm{mm}$ \\
\hline Refractive camera & $f / 2.94$, focal length $=455 \mathrm{~mm}$ \\
\hline Detector & $\mathrm{CCD} 22 \mathrm{v} 231-84,4 \mathrm{k} \times 4 \mathrm{k}, 15 \mu \mathrm{m} / \mathrm{pix}$ \\
\hline Operating temperature & $285 \mathrm{~K}$ \\
\hline
\end{tabular}

Table 1. Main features of CARMENES VIS spectrograph.

\section{RAMSES - A SPECTROGRAPH PHYSICAL MODELING SOFTWARE}

We present RAMSES - RAy tracing and Modelling Software for Echelle Spectrographs -, a ray tracing software that computes the centroid position on the detector of any spectral feature defined by its wavelength. We employ an object-oriented philosophy in which each optical element is represented by an independent module that describes its optical surfaces and how rays are traced through it.

A high resolution echelle spectrograph's most basic layout consists of a slit, a collimator, a high dispersion element, a cross-dispersion element, a camera and a detector. In the case of CARMENES, the slit is an octagonal fiber, the collimator is a parabolic mirror, the main dispersing element an echelle grating, the cross-dispersion element is a grism, the camera an array of 5 lenses plus a field flattener, and the detector a CCD. In addition, the CARMENES optical design includes an FN-system, which transforms the output beam of the octagonal fiber to the working F-number of the collimator, and a fold mirror, which redirects the intermediate focus image plane beam back to the collimator, as shown in fig. 2 .

The modules that describe the optical elements of the spectrograph are then: slit, F/N system, collimator, echelle grating, transfer mirror, grism, camera lens and detector. They are defined by a set of attributes related to the physical properties of each element. 

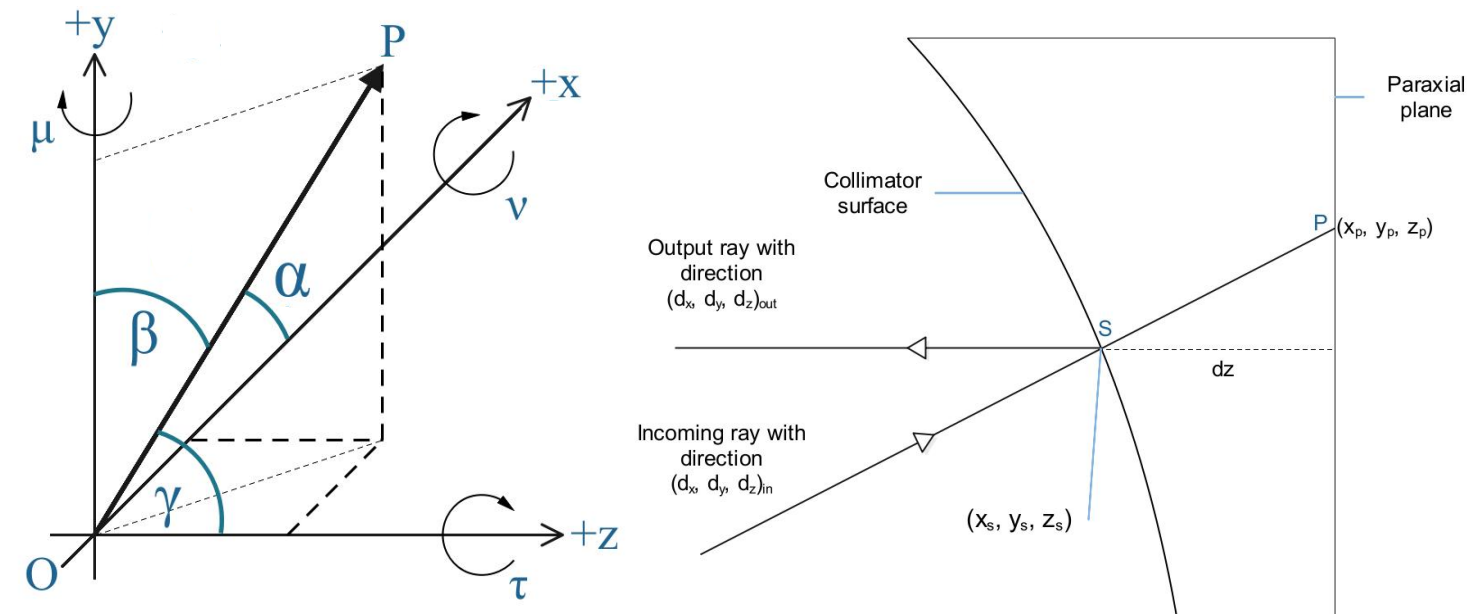

Figure 2. Left: Coordinate system orientation. Direction angles $(\alpha, \beta, \gamma)$ are positive from axis to ray OP. Rotation angles around xyz-axis are given by $\mu, \nu$ and $\tau$, respectively. Optical axis is along the z-axis, echelle dispersion direction along $\mathrm{x}$-axis and cross-dispersion direction along $\mathrm{y}$-axis. Right: $2 \mathrm{D}$ projection of the collimator.

\subsection{Coordinate system}

Coordinates are described by the position vector $\vec{x}=[x, y, z]$ and the direction cosines $\vec{d}=\left[d_{x}, d_{y}, d_{z}\right]$ in a three dimension Euclidean space, so

$$
\cos ^{2} \alpha+\cos ^{2} \beta+\cos ^{2} \gamma=d_{x}^{2}+d_{y}^{2}+d_{z}^{2}=1
$$

where $\alpha, \beta$ and $\gamma$ are the direction angles. The direction angles are acute, i.e., $0<\alpha<\pi, 0<\beta<\pi$ and $0<\gamma<\pi$, and they denote the angles between $\vec{x}$ and the unit basis vectors.

Each ray is defined by its wavelength $\lambda$, position $\vec{x}$ and direction $\vec{d}$. The optical axis is along the z-axis, the $\mathrm{x}$ axis is along the echelle-dispersion direction and $\mathrm{y}$ is along cross-dispersion direction. Angles are measured positive from axis to ray, as shown in fig. 2 . Each module receives as input a vector of the form $(\lambda, \vec{x}, \vec{d})$. The input position corresponds to the intersection of the ray at the paraxial plane with the first surface of the optical element, and the module computes the position and direction of the ray after passing through it.

Each ray goes from one optical element to another according to the free space ray tracing equation. Tilts are applied using rotation matrices of the form

$$
R_{\mu / x}=\left(\begin{array}{ccc}
1 & 0 & 0 \\
0 & \cos \mu & -\sin \mu \\
0 & \sin \mu & \cos \mu
\end{array}\right), R_{\nu / y}=\left(\begin{array}{ccc}
\cos \nu & 0 & -\sin \nu \\
0 & 1 & 0 \\
\sin \nu & 0 & \cos \nu
\end{array}\right), R_{\tau / z}=\left(\begin{array}{ccc}
\cos \tau & -\sin \tau & 0 \\
\sin \tau & \cos \tau & 0 \\
0 & 0 & 1
\end{array}\right)
$$

where rotations around the $\mathrm{x}, \mathrm{y}$ and $\mathrm{z}$ axis are given by $R_{\mu / x}, R_{\nu / y}$ and $R_{\tau / z}$, respectively. The overall rotation matrix is given by the multiplication of each rotation matrix in the following order $R=R_{\mu / x} \cdot R_{\nu / y} \cdot R_{\tau / z}$.

\subsection{Ray tracing and exact corrections for curved surfaces}

The collimator and the camera consist of reflective and refractive curved surfaces. The collimator is a paraboloid, while the camera is composed of 5 spherical lenses plus a field flattener with a toroidal output surface. In total we consider three types of curved surface types: parabolic, spherical and cylindrical. In order to trace the ray path along the spectrograph we must calculate exactly the position where it hits the surface to calculate the normal vector in this position. Given the position, the normal vector and the properties of the surface, we can calculate the output direction of the ray after a curved surface. Let's consider the ray entering the curved surface shown in the right panel of fig. 2. The beam with incoming direction $\vec{d}_{\text {in }}$ is reflected by the collimator mirror to an output direction $\vec{d}_{\text {out }}$. If $\left(x_{p}, y_{p}\right)$ are the coordinates at the paraxial plane of the surface, and $\left(x_{s}, y_{s}\right)$ are the coordinates at the surface, then the direction tangents of the ray are given by

$$
\frac{d_{x}}{d_{z}}=\frac{x_{s}-x_{p}}{d z}, \quad \frac{d_{y}}{d_{z}}=\frac{y_{s}-y_{p}}{d z}
$$


where $d z$ is the distance along the optical axis from the paraxial plane to the surface. On the other side, a curved surface with curvature radius $r$ is described by its sag equation, which is given by

$$
\text { sag }= \begin{cases}x^{2}+y^{2}+(z-r)^{2}=r^{2}, & \text { spherical } \\ x^{2}+y^{2}=2 r z, & \text { parabolic } \\ x^{2}+(z-r)^{2}=r^{2}, & \text { cylindric } \\ x^{2}+y^{2}=r z\left(1+\sqrt{1-(1+\kappa) \frac{\left(x^{2}+y^{2}\right)}{r^{2}}}\right), & \text { aspheric }\end{cases}
$$

Using eqs. 3 and 4 we can calculate $d z$ and the exact position of the ray at the optical surface as a function of the input ray direction and the position on the paraxial plane. With the exact position we obtain the normal vector. For a reflective surface the new direction is

$$
d_{\text {new }}=d_{\text {old }}-2 \cos \theta \cdot \vec{n}
$$

where $\theta$ is the angle between the direction and the normal vector.

For a refractive surface the output direction is given by Snell's law

$$
d_{\text {new }}=\frac{d_{\text {old }}}{k}-\left(\cos r-\frac{\cos i}{k}\right) \cdot \vec{n}
$$

where $k=n_{1} / n_{0}$ and $n_{0}$ and $n_{1}$ are the refractive indices before and after refraction, respectively. $i$ and $r$ are the incident and refracted angles with respect to the normal vector $\vec{n}$. Using eqs. 3 to 6 we can trace exactly a ray through a curved surface.

\subsection{Optical elements modules}

\subsubsection{Slit}

The entrance slit is defined by its position $\vec{x}=(x, y, z)$ and angles of orientation $\mu_{\text {slit }}, \nu_{\text {slit }}$ and $\tau_{\text {slit }}$.

\subsection{2 $\mathrm{F} / \mathrm{N}$ system}

The $\mathrm{F} / \mathrm{N}$ system is defined by the curvature radii of the surfaces, and the materials and thicknesses of the lenses used. It is composed of two spherical lenses. We trace from the fiber plane to the paraxial plane of the FN-system, and then through the rest of the system using the equations from section 3.2.

\subsubsection{Collimator}

The collimator is a parabolic mirror defined by its curvature and conic constant. The input to this module are the coordinates at the paraxial plane of the collimator and the direction of the incoming ray. The output are the position of the ray at the surface of the mirror and the direction of the ray after the reflection on the mirror's surface. Given the position of a ray in the paraxial plane and using equations from section 3.2, we calculate the output direction from the collimator.

\subsubsection{Echelle grating}

The echelle grating is defined by the grating constant $G$ and the orientation of the grating. Following the generalized ray-tracing approach from Mitchell $1981,{ }^{9}$ the directions of the diffracted rays are given by

$$
\begin{array}{r}
d_{x, \text { out }}=d_{x, \text { in }} \\
d_{y, \text { out }}=\frac{m \cdot \lambda}{d}-d_{y, \text { in }} \\
d_{z, \text { out } t}=\sqrt{1-d_{x, \text { out }}^{2}-d_{y, \text { out }}^{2}}
\end{array}
$$

where $m$ is the diffraction order, $\lambda$ the wavelength and $d$ the groove spacing of the grating, which is related to the grating constant according to $d=1 / G$. We assume that the refractive indices before and after the grating are the same. Eqs. 7 describe diffraction on a reflection grating. As no simple optical equation allows to derive the z-component of the direction vector, we derive it from the normalization relation. 


\subsubsection{Folding mirror}

The folding mirror is a flat mirror and is described by its position and orientation. The output direction of the traced rays are determined by the law of reflection given by eq. 5 .

\subsubsection{Grism}

The cross-dispersing element is a grism. A grism is a prism with a transmission grating as an output surface. It is defined by the prism apex angle, the grating constant of the output surface, the base thickness, position and orientation angles. The grism module evaluates the Snell's law at the input surface using eq. 6. We trace from the input to the output surface given the base thickness of the grism. Once at the output surface we apply Snell's law to refract the rays due to the glass to air interface and we calculate diffraction angles at the grating surface. The output surface of the grism acts as a transmission grating, thus diffraction is given by

$$
\begin{array}{r}
d_{x, \text { out }}=\frac{m \cdot \lambda}{d_{G}}+d_{x, \text { out }} \\
d_{y, \text { out }}=d_{y, \text { in }} \\
d_{z, \text { out }}=\sqrt{1-d_{x, \text { out }}^{2}-d_{y, \text { out }}^{2}}
\end{array}
$$

where $d_{G}$ is the groove spacing of the grism output surface, $m$ is the diffraction order, which in our case is $m=1$, and $\lambda$ is the wavelength.

\subsubsection{Camera}

The camera module performs the ray tracing through a multi-element optical system that focuses the spectrum on the detector. The CARMENES VIS camera consist of five lenses plus a field flattener. All lenses are "airspaced" due to the fact that the instrument is working in vacuum. The field lens acts as entrance window to the detector head dewar.

After the grism output surface we trace each ray to the paraxial plane of the first surface of the first lens of the camera. These are the input coordinates to this module. For each surface of the camera we calculate the exact position of the intersection between the ray and the surface, and we trace the rays following the procedure described in section 3.2. The field flattener is mechanically separated from the rest of the camera and is mechanically connected to the detector. Because of this, we treat the first five optical elements of the camera as one element (CAM1), and the field flattener (FF) as another. The output from CAM1 is then traced through the FF to the detector plane. Because of this, decentering and tilts are independent in CAM1 and FF.

All surfaces are spherical, except for the last surface of the FF which is toroidal. For simplicity we approximate the toroidal surface with a cylindrical surface, described by eq. 4 .

\subsubsection{Detector}

The detector is described by the number of pixels in the $\mathrm{x}$ and $\mathrm{y}$ directions, the pixel size, position and orientation. The main features for the CARMENES detector can be found in Table 1.

\subsubsection{Materials and environment}

The path that a photon will follow inside an optical element depends on the properties and the chemical composition of the glass material. In CARMENES, the grisms and the lenses of the objective camera are made of a variety of materials from Ohara and Schott catalogs. In particular, the VIS channel uses LF5 for the grism and S-FPL51, S-FPL53, S-BAM4, S-BSL7, S-LAL10 and SILICA for the camera. Glass manufacturer catalogs provide refractive indices for the different materials with an accuracy of $\pm 3 \mathrm{e}-5$, and we use those reference values to compute the refractive index for a given wavelength under the operating conditions of the spectrograph. The instrument operates in vacuum conditions and at a stable temperature of $12^{\circ} \mathrm{C}$ in the VIS channel. 


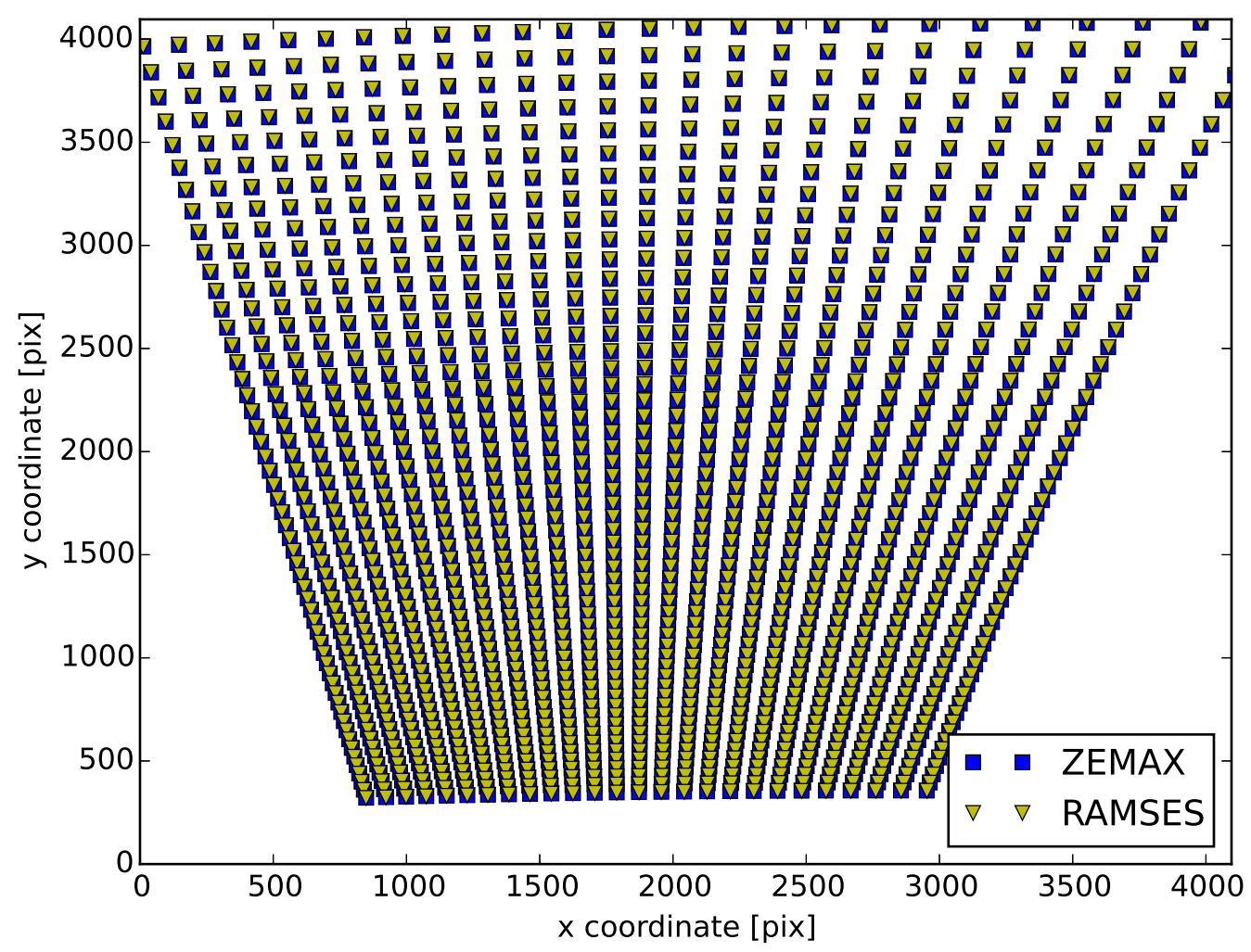

Figure 3. Echellogram traced by Zemax (blue squares) and RAMSES (yellow triangles).

\subsection{Comparing with ZEMAX}

We compared RAMSES with the commercial ray tracing software ZEMAX. For a list of wavelengths, we performed ray tracing through the spectrograph using our model and ZEMAX. We evaluate the performance of our model by calculating the rms of the difference between the $\mathrm{x}$ and $\mathrm{y}$ positions at the detector focal plane with respect to Zemax, resulting in a rms of 1/1000 of a pixel . Fig. 3 shows the echellogram traced with our model in yellow triangles and traced by Zemax in blue squares. Both models coincide up to a precision of a small fraction of a pixel.

RAMSES offers more flexibility when modifying the source code to implement additional functionalities, and it runs three orders magnitude faster than Zemax, making it ideal for performing optimization routines that require a large number of calculations.

\section{FITTING CARMENES SPECTRA}

\subsection{Spectral features selection}

We tested our model against HCL exposures obtained with the CARMENES VIS and NIR spectrographs. The wavelength calibration strategy for CARMENES is based on the use of HCL spectra to create a wavelength solution to calibrate the Fabry-Perot spectrum that is observed simultaneously with science observations to track for instrumental drifts. The wavelength solution is modeled with polynomials in direct regression with $x(\lambda)=\operatorname{poly}(o, \lambda),{ }^{10}$ where $x$ is the position of a spectral feature on the detector in pixels along the echelle dispersion axis, $o$ the echelle order number and $\lambda$ the wavelength. The VIS channel uses three different HCLs: Th-Ne, U-Ar and U-Ne, while the NIR channel uses a U-Ne lamp. We use the Redman et al. $2014^{11}$ catalog for the Th lines, Sarmiento et al. $2016^{12}$ for the U lines, and the NIST Atomic Spectra Database ${ }^{13}$ for the Ne and 


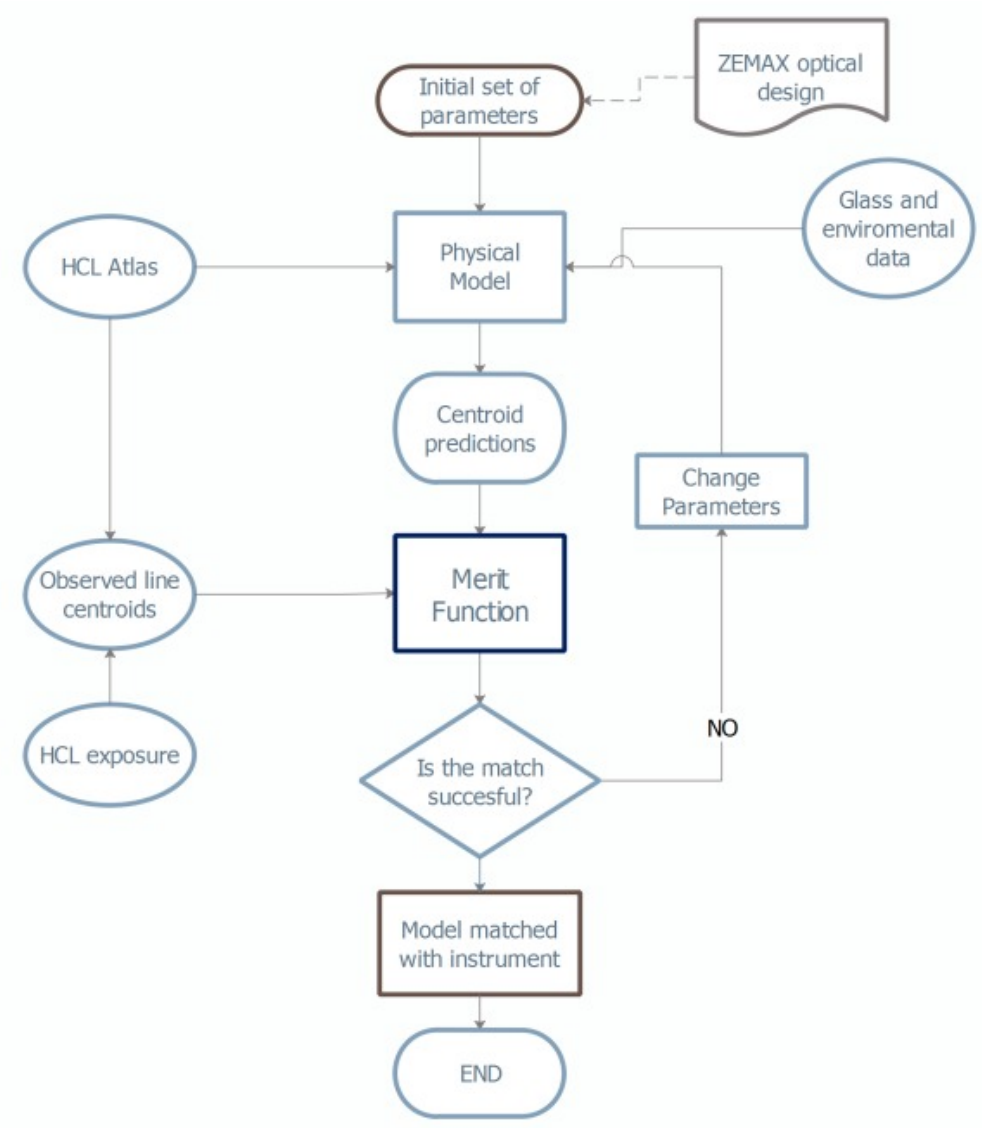

Figure 4. Flowchart of model optimization algorithm to match with instrument observations. Credit: Bristow et al. 2008. ${ }^{14}$

Ar lines. Close to 10,000 lines are identified in the lamp spectra of the VIS spectrograph, but only a fraction of them are used for fitting our model. We chose all lines with measured positions with an error less than 0.005 pixels, resulting in a sample of 1,154 spectral lines.

\subsection{Optimization algorithm and parameter choice}

To fit a physical model to the instrument, we employ an optimization routine. Fig. 4 shows the flowchart of the procedure. Due to the nature and complexity of the model, there are degeneracies in the solutions, e.g. a tilt in the y-direction in the transfer mirror can be compensated by a y-tilt of the collimator in the opposite direction and still provide a good solution for two different set of parameters. To ensure that we find the global minimum of the function, we employ a Simulated Annealing (SA) algorithm to search for the parameters that best fit the data. The annealing procedure is analogous to as tempering certain alloys of metal, glass or crystal by heating above its melting point, holding its temperature, and then cooling it down very slowly until it solidifies. The defect-free crystal state corresponds to the global minimum energy configuration. The physical material states correspond to the problem solutions, the energy of a state to cost of the solution, and the temperature to a control parameter. We chose $S A$ as it can deal with highly non-linear models, and one of its main advantages is its ability to approach the global minimum without getting stuck in a local one. It is also quite versatile since it does not rely on any restrictive properties of the model. Two of its weaknesses are that one has to take into account the different types of constraints of the model, and that there is a clear trade-off between the quality of the solutions and the time required to compute them.

The model parameter space consist of 45 parameters, describing the physical properties of the spectrograph. 

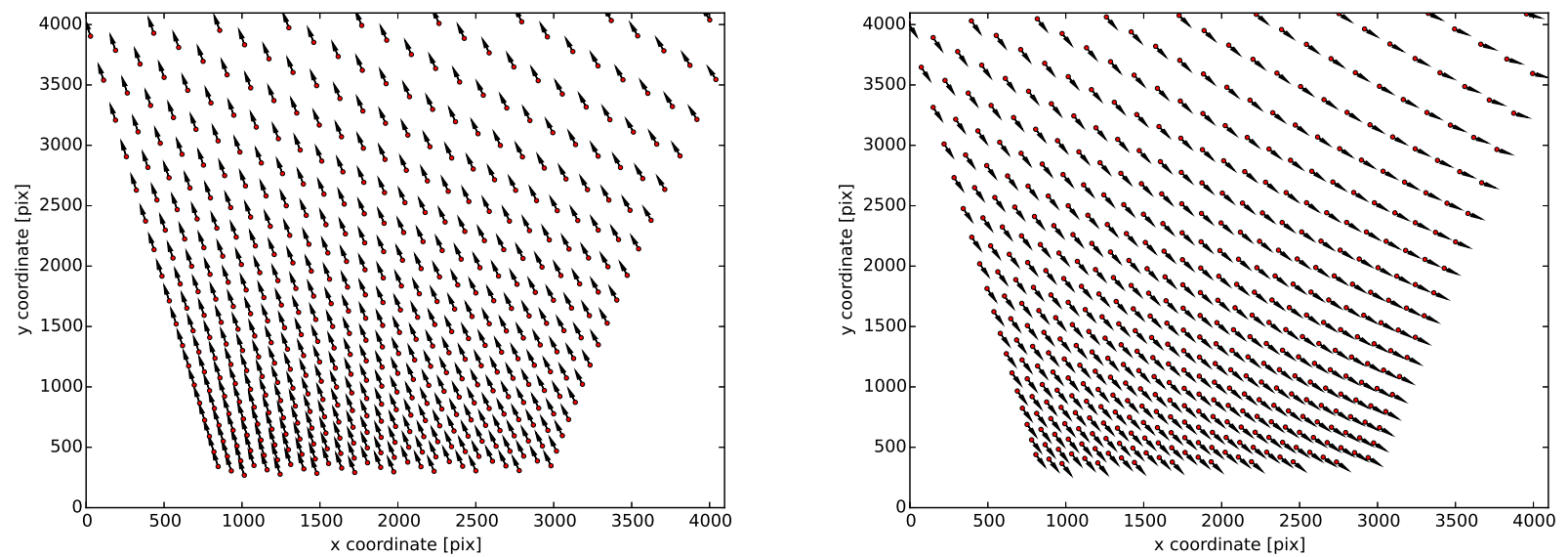

Figure 5. Vector maps for two different parameter sets. The red points are the original positions of the spectral lines selected, while the black arrows represent how much each spectral line has been shifted for the two different sets of parameters used.

From these 45 parameters 22 are left open, which are: slit orientation and position, slit-collimator distance, collimator x-y tilt angles, echelle orientation angles, collimator-transfer mirror distance, transfer mirror x-y tilts, grism orientation angles, camera to field flattener distance, field flattener-CCD orientation angles, CCD position and orientation angles. The centroids of the selected features are used to optimize the open parameters to match with the model predictions. We studied the effect of each parameter in the detector plane using patterns in vector maps showing the difference between the design and the perturbed mapping of the spectral line positions. Fig. 5 shows two examples of vector maps for two different configurations where we have changed the value of some of the parameters by a small amount. The final shifts are a combined effect of all the parameters. Selecting subsets of parameters and studying their residuals provides a better understanding of the nature of the parameters used to fit our model to the observations.

\subsection{Preliminary Results}

We performed the simulations while the instrument was already in operation. We obtained the data for the positions of the spectral lines on the detectors from the CARMENES data archive. We start with an initial guess based on the design parameters of the spectrograph from ZEMAX. Fig. 6 shows the match between our model and an observation, for the set of wavelengths used for optimization. The left panel in fig. 6 , shows a section of a U-Ar spectrum. On top of it we plot the line positions derived by the CARMENES reduction pipeline, together with the wavelength solution calculated by RAMSES, for the two fibers that feed the spectrograph. As the line list we are using contains spectral lines from Th-Ne, U-Ar and U-Ne HCLs, some of the positions from the catalog don't match the image in fig. 6, as the spectra we show in this figure corresponds to a U-Ar spectrum. However, the positions for the $\mathrm{U}$ and Ar lines are in agreement with expectations.

The right panel in fig. 6 shows the wavelength solution. The blue points correspond to the x-position in pixels of the selection of spectral lines used to fit our data, while the black lines correspond to the wavelength solution calculated by RAMSES.

We achieve sub-pixel accuracy by optimizing the different parameters. The residuals are shown in the left panel in fig. 7. The scatter of the residuals seems to concentrate around the center, but we observe a gradient in the residuals of the y axis. This might be related to a parameter that could require special weighting when optimizing. However, due to the strong degeneracies in the parameter space it is not simple to disentangle the particular effect of one parameter on the residuals, as they are strongly correlated. The right panel of fig. 7 shows the residuals between the measured positions of spectral lines and the prediction from our model. The square indicates one pixel of the detector. We observe an asymmetry in the distribution, however without a detailed analysis of the line centering method it is not possible to relate the asymmetry to systematics in either 

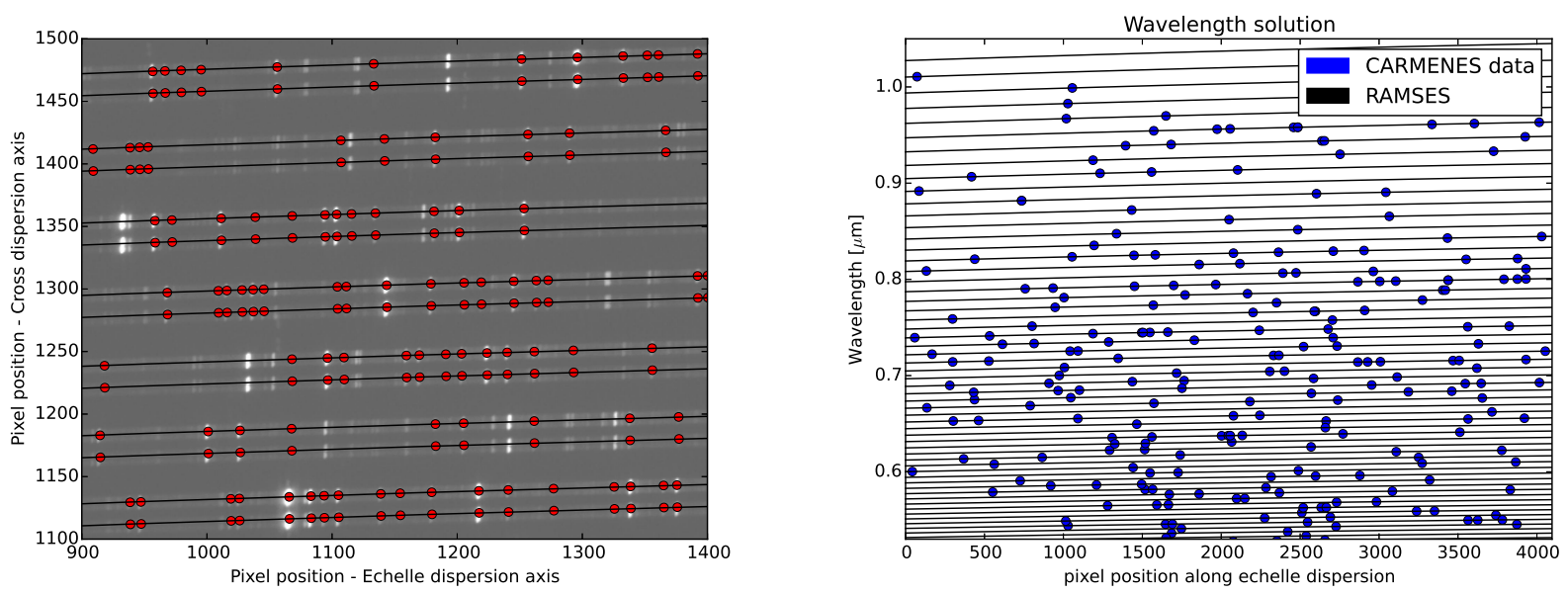

Figure 6. Left: The red points corresponds to the measured positions of the selected spectral features on the CCD across different orders of a U-Ar lamp spectra. The black line is the RAMSES prediction for the position of the wavelengths along the echelle dispersion direction. Right: Wavelength solution $x=x(\lambda)$ computed with RAMSES. The $\mathrm{x}$-axis corresponds to the spectral line position along the echelle dispersion direction and the $y$-axis is the wavelength of that particular feature.
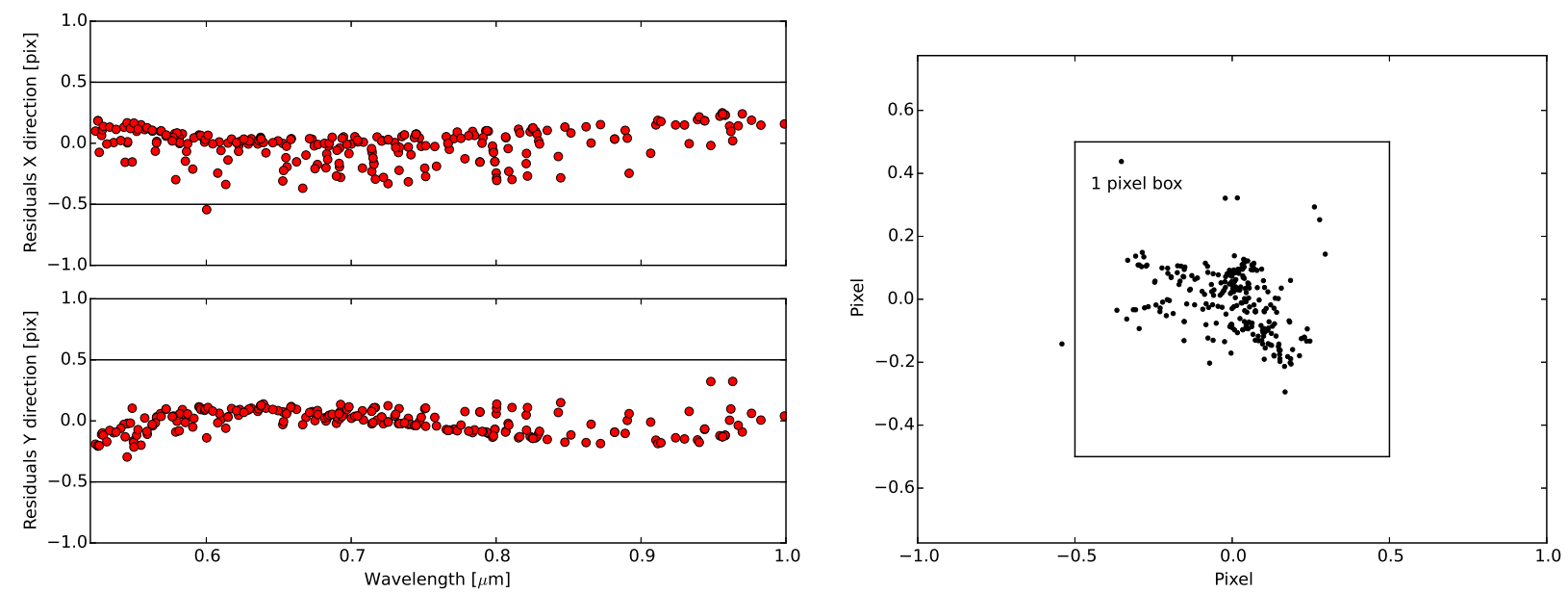

Figure 7. Left: Residuals in echelle and cross dispersion direction for a sample of spectral lines. Right: Scatter of residuals. The box represents one pixel.

the model or the measurements. Our model currently fits all the lines with an accuracy better than half a pixel.

\section{SUMMARY}

We presented RAMSES, a ray tracing software to model high resolution echelle spectrographs. We tested our modeling scheme using calibration data available in the CARMENES database. We have shown that RAMSES can compute the wavelength solution with sub-pixel accuracy. Using a reduced number of spectral lines, spread all over the detector, we can predict the position of any wavelength on the detector.

The implementation of this modeling scheme does not depend on any commercial optical design software. However, we have used ZEMAX to compare the performance of RAMSES and some of the design parameters are extracted directly from ZEMAX.

The most direct application of this instrument model is in the area of data calibration. For low and high resolution spectroscopy, RAMSES can be a useful tool to provide a purely-instrumental wavelength solution, without 
the need of polynomial fitting or other techniques. However when working in the high precision RV regime, our residuals must be reduced considerably, in order to compete with current wavelength solution computations that provide an accuracy of the order of $\sim 0.001$ pixel. This could be achieved by adding a low-order polynomial or other function designed to capture system aberrations.

The combination of modeling techniques and calibration data can be used to optimize instrument performance throughout the instrument lifetime. The key to achieve the best science product is an integrated approach that combines the development of physical instrument models and the application of the model during integration, testing, commissioning and science verification, as well as the integration of the model in the data reduction pipeline.

Further improvements in RAMSES aim at reducing the residuals to a thousandth of a pixel, so that the solution is accurate enough to compute high precision radial velocities.

\section{ACKNOWLEDGMENTS}

We would like to thank our colleagues Dr. Julian Stürmer and Dr. Robert Harris for their support and contribution to this work providing ideas, feedback and very useful discussions.

\section{REFERENCES}

[1] Ballester, P. and Rosa, M. R., "Modeling echelle spectrographs," Astronomy and Astrophysics Supplement Series 126, 563-571 (1997).

[2] Rosa, M. R., Alexov, A., Bristow, P., and Kerber, F., "Fos post-operational archive and stis calibration enhancement," The 2002 HST calibration workshop (2002).

[3] Kerber, F., Bristow, P., and Rosa, M. R., "Stis calibration enhancement (stis-ce): Dispersion solutions based on a physical instrument model," The 2005 HST calibration workshop (2005).

[4] Bristow, P., Kerber, F., Rosa, M. R., Pirard, J. F., Siebenmorgen, R., and Kaufl, H., "Model-based wavelength calibration for crires," Proc. SPIE 6270 (2006).

[5] Bristow, P., Vernet, J., Kerber, F., Moehler, S., and Modigliani, A., "Using the x-shooter physical model to understand instrument flexure," Proc. SPIE 7735 (2010).

[6] Chanumolu, A., Jones, D., and Thirupathi, S., "Modelling high resolution echelle spectrographs for calibrations: Hanle echelle spectrograph, a case study," Experimental Astronomy 39, 423-443 (2015).

[7] Chanumolu, A., Thirupathi, S., Jones, D., Giridhar, S., Grobler, D., and Jakobsson, R., "Performance results of HESP physical model," Experimental Astronomy 43, 39-58 (Feb. 2017).

[8] Quirrenbach, A. and CARMENES-Consortium, "Carmenes: an overview six months after first light," (2016).

[9] Mitchell, C. J., "Generalized ray-tracing for diffraction gratings of arbitrary form," Journal of Optics 12(5), 301 (1981).

[10] Bauer, F. F., Zechmeister, M., and Reiners, A., "Calibrating echelle spectrographs with fabry-perot etalons," Astronomy and Astrophysics 117 (2015).

[11] Redman, S. L., Nave, G., and Sansonetti, C. J., "The spectrum of thorium from 250nm to 5500nm: Ritz wavelengths and optimized energy levels," The Astrophysical Journal Supplement Series 211(1), 4 (2014).

[12] Sarmiento, L. F., Reiners, A., Seemann, U., Lemke, U., Winkler, J., Pluto, M., Gnther, E. W., Quirrenbach, A., Amado, P. J., Ribas, I., Caballero, J. A., Mundt, R., and Seifert, W., "Characterizing u-ne hollow cathode lamps at near-ir wavelengths for the carmenes survey," (2014).

[13] Kramida, A., Ralchenko, Y., Reader, J., and NIST-ASD-Team-(2015), "Nist atomic spectra database (ver. 5.3)," (2016).

[14] Bristow, P., Kerber, F., and Rosa, M. R., "Advanced calibration techniques for astronomical spectrographs," The Messenger 131 (2008). 